\title{
Relaciones entre atención conjunta y desarrollo del vocabulario en niños con síndrome de Down
}

\author{
Miguel Galeote, Elena Checa, Concepción Sánchez y Antonio Gamarro
}

Universidad de Málaga, España

\begin{abstract}
Resumen
La atención conjunta (AC) es considerada uno de los prerrequisitos para el desarrollo lingüístico. Curiosamente, dicha AC no ha sido ampliamente estudiada en los niños con síndrome de Down (SD). Además, las investigaciones realizadas muestran resultados poco claros. Nuestro objetivo es analizar la relación entre la AC y el desarrollo del vocabulario en una amplia muestra de niños con SD. En el estudio, participaron 131 niños con SD y 131 niños con desarrollo típico (DT) entre los 8 y los 29 meses de edad de desarrollo. Se empleó el Inventario de Desarrollo Comunicativo MacArthur-Bates (CDI) adaptado a los niños con SD. Los ítems relativos a la AC fueron categorizados en función del grado de habilidad. Los resultados muestran bastante similitud entre los niños de ambos grupos en todas las variables examinadas. La diferencia más significativa tenía que ver con la superioridad de los niños con $\mathrm{SD}$ en la comprensión. No se encontró un efecto diferencial entre las conductas protoimperativas y protodeclarativas.

Palabras clave: Atención conjunta; Desarrollo del vocabulario; síndrome de Down.

\section{Relationship between joint attention and vocabulary development in Spanish children with Down syndrome}

\begin{abstract}
Joint attention (JA) has been regarded as a pre-requisite for language development. Curiously, JA has not been widely studied in children with Down syndrome (DS). Moreover, the research carried out does not show very clear results. Our aim is to evaluate the relationship between JA and vocabulary development in a wide sample of children with DS. A total of 131 children with DS and 131 children with typical development took part in the study, aged between 8 and 29 months of mental age. An adapted version for children with DS of MacArthur-Bates Communicative Development Inventory was used. Items related to JA were categorized according to the skill level. The results from both groups of participants were similar in all the variables examined. The most remarkable difference had to do with a better performance of children with DS in comprehension measures. No differences were found in protoimperative and protodeclarative responses.
\end{abstract}

Key words: Down syndrome; Joint attention; Lexical development.

Correspondencia con los autores: mgaleote@uma.es

Recibido 26 de Mayo de 2013. Aceptado 02 de Julio de 2013

Reconocimiento y agradecimientos: Investigación financiada por el Ministerio de Ciencia e Innovación (Dirección General de Programas y Transferencia de Conocimiento) y FEDER (Proyecto: PSI200802748). Expresamos nuestro agradecimiento a todos los niños y familias, los numerosos terapeutas que trabajan en las Asociaciones de personas con síndrome de Down y Centros de Atención Temprana, así como al personal de las guarderías y Centros de Educación Infantil que han participado en este trabajo. 


\section{Introducción}

La atención conjunta $(\mathrm{AC})$ la habilidad para coordinar la propia atención con la de un socio comunicativo en relación con un objeto o evento (Carpenter, Nagel y Tomasello, 1998). La AC supone, por tanto, un intercambio triádico (Bakeman y Adamson, 1986). Este patrón de interacción triádica pone de manifiesto la emergente comprensión del bebé de que las personas tienen puntos de vista independientes que pueden ser compartidos (Sigman y Ruskin, 1999) y es coincidente con la noción de intersubjetividad secundaria de Trevarthen (1998).

Las conductas de AC pueden ser usadas para funciones imperativas y declarativas (Bates, Benigni, Bretherton, Camaioni y Volterra, 1979), en situaciones de iniciación de propuestas de interacción a los socios comunicativos o de respuesta a las propuestas de estos (Seibert, Hogan y Mundy, 1982). De este modo, la AC puede analizarse en varios componentes (Seibert et al., 1982): (1) iniciar peticiones / regulaciones de la conducta: habilidad para usar la mirada y gestos con el fin de pedir ayuda a la otra persona para obtener un objeto o evento (protoimperativos), (2) iniciar conductas de AC: habilidad para usar la dirección de la mirada y gestos con el fin de dirigir la atención de otras personas para compartir experiencias (protodeclarativos), (3) responder a las peticiones de otras personas: habilidad para responder a una orden como "dámelo" presentada con un gesto y (4) responder a conductas de AC: habilidad para seguir la dirección de la mirada y atender a los gestos de otras personas. Cada uno de estos componentes implica habilidades específicas.

De especial importancia son los actos declarativos en los que el niño comparte una experiencia o conciencia de un objeto o evento (Bates et al., 1979). Para algunos autores (p. ej. Tomasello, 1995), estos actos pondrían de manifiesto que los niños perciben a otras personas como agentes intencionales puesto que comprenden que esas personas tienen estados mentales internos tales como atención, intereses, deseos e intenciones y que estos estados internos pueden ser compartidos y / o actuar sobre ellos.

Aunque no todas las conductas de AC surgen al mismo tiempo, suele estimarse que hacia los 12 meses la mayoría de los niños con desarrollo típico (DT) muestran todos los aspectos de la AC, incluyendo el compartir la atención (por ejemplo, a través del uso de la alternancia en la mirada), seguir la atención del otro (siguiendo la dirección de la mirada o un gesto) y dirigir la atención del otro (Carpenter et al., 1998). El uso del lenguaje en la interacción sería más tardío.

En los últimos años, ha ido surgiendo un interés creciente por el estudio de la 
AC. Ello se debe a las implicaciones que ésta tendría en relación con el desarrollo cognitivo y social, así como con el desarrollo del lenguaje. Por lo que respecta al desarrollo del lenguaje, numerosos trabajos han encontrado una relación entre éste y las habilidades de AC en niños con DT (Akhtar, Dunham y Dunham, 1991; Harris, Jones, Brookes y Grant, 1986; Mundy y Gomes, 1998; Tomasello y Farrar, 1986; Tomasello y Todd, 1983). Diversos estudios, por otro lado, han encontrado diferentes efectos de las conductas de iniciación de / respuesta a la AC en el desarrollo del lenguaje. Mundy, Kasari, Sigman y Ruskin (1995), por ejemplo, encontraron una asociación entre las conductas interactivas sociales activas (elicitar la atención de otra persona, implicarse en la toma de turnos con objetos, etc.) y el lenguaje expresivo. Mundy y Gomes (1998) encontraron que las conductas de iniciación de la AC (contacto ocular, mostrar y apuntar a los objetos) también estaban asociadas con el lenguaje expresivo. Esto se corresponde con la visión de Bates, Thal, Fenson, Whitesell y Oakes (1989) quienes asumen que las habilidades de interacción social y el lenguaje expresivo comparten una varianza común en el área de la motivación social y la iniciación de conductas sociales. Por otro lado, otros estudios indican que el seguir la AC (responder a ella) correlaciona más con la comprensión del lenguaje (Laakso, Poikkeus, Katajamäki y Lyytinen, 1999, Mundy y Gomes, 1998).

Por lo que respecta a los niños con síndrome de Down (SD), su desarrollo de la $\mathrm{AC}$ ha sido relativamente estudiado, aunque la mayoría de los trabajos incluyen muestras bastante pequeñas. Además, algunos de los resultados encontrados son contradictorios. Según algunos autores, los niños con SD adquieren habilidades de AC (Sigman y Ruskin, 1999), producen signos referenciales (Franco y Wishart, 1995), miradas de AC coordinada (Kasari, Freeman, Mundy y Sigman, 1995) y lentamente van siendo capaces de implicarse en episodios de AC coordinada durante las interacciones sociales (Legerstee y Weintraub, 1997). Sin embargo, en otros estudios se han encontrado algunas limitaciones en el desarrollo de la $\mathrm{AC}$ en los niños con SD. Estas limitaciones tienen que ver con el modo en que se implican con los objetos, con el tipo de señales sociales que producen y con el estilo interactivo de los cuidadores.

Por lo que respecta a su relación con los objetos, algunos estudios han mostrado que, en comparación con los niños con DT, los niños con SD presentan una implicación menos activa con los juguetes (Krakow y Koop, 1983; MacTurk, Vietze, McCarthy, McQuiston y Yarrow, 1985). Legerstee y Weintraub (1997 -ver también Landry y Chapieski, 1989) también encontraron que los bebés con SD dedicaban menos tiempo 
que los bebés con DT al juego con objetos, así como a coordinar su atención entre las personas y los objetos. Por el contrario, los bebés con SD dedicaban más tiempo a compartir pasivamente la atención a los objetos con los adultos. Mundy, Sigman, Kasari y Yirmiya (1988) encontraron que los niños con SD encuentran más problemas a la hora de pedir objetos o ayuda para conseguirlos. Según esto, pueden presentar más problemas a la hora de sostener la $\mathrm{AC}$ en contextos relativos a peticiones.

Por lo que respecta a las señales sociales, varios estudios, empleando niños con SD y con DT emparejados en EM, han encontrado que los niños con SD generan menos señales apropiadas de este tipo (Fischer, 1987; Mundy et al., 1988; Tannock, 1988), muestran menor capacidad de respuesta a las señales de sus madres (Beeghly, Perry y Cicchetti, 1989; Maurer y Sherrod, 1987; Tannock, 1988) y un número menor de patrones predecibles de respuesta (Cielinski, Vaughn, Seifer y Contreras, 1995; Landry y Chapieski, 1989; Legerstee y Bowman, 1989).

Por último, se ha propuesto que la falta de atención de los bebés con SD a los objetos y sus dificultades en el dominio de lo social dificultarían el establecimiento de la $\mathrm{AC}$ con sus cuidadores. Más concretamente, se propone que, en ausencia de señales claras y frecuentes por parte de sus hijos, las madres de los bebés con SD adoptan un estilo más directivo y controlador que las madres de niños con DT (Cardoso-Martins y Mervis, 1985; Davis, Stroud y Green, 1988; Mahoney, Fors y Wood, 1990). En este sentido, algunos trabajos han encontrado que las madres de niños con SD son más directivas y controladoras (Cardoso-Martins y Mervis, 1985; Mahoney et al., 1990; Tannock, 1988). En los estudios que se ha investigado la atención de los niños a los juguetes dentro del contexto de interacción madre-niño, también se ha encontrado tasas más elevadas de cambio de tópico y mayor control sobre el tópico en las madres con niños con SD que en madres con niños con DT (Mahoney et al., 1990; Tannock, 1988). También se ha observado que las madres de niños con SD cambian de tópico tanto cuando sus niños están implicados en los objetos como cuando no lo están (Mahoney et al., 1990; Maurer y Sherrod, 1987; Tannock, 1988). En muestras de niños con DT, los cambios de tópico maternos parecen ir en consonancia con el curso de la interacción (Tannock, 1988).

Como consecuencia de todo lo anterior, los niños con SD podrían experimentar menor cantidad de AC que los niños con DT. De hecho, la interrupción de la AC podría ocurrir más frecuente en díadas de niños con síndrome de Down y sus cuidadores. Todo ello podría retrasar su desarrollo del lenguaje. 
El objetivo del presente trabajo consiste en analizar la relación entre diferentes conductas de AC y el desarrollo del vocabulario en una amplia muestra de niños con SD y niños con DT. Para tal fin, se ha considerado las respuestas de informes paternos relativas a conductas de AC en: (1) el Inventario de Desarrollo Comunicativo MacArthur-Bates adaptado para niños con SD (Galeote, Soto, Lamela, Checa, Pulido y Rey, 2006) y (2) la adaptación realizada por Hernández (1995) del cuestionario sobre "evaluación de la comunicación" (ECO) (ver apartado de materiales en método). En ambos casos, sólo se han tenido en cuenta las conductas de iniciación de AC. A cada niño se le adjudicó una puntuación considerando la distinta complejidad de las conductas de AC realizadas. Por otro lado, con el fin de analizar la relación entre el nivel de AC y el desarrollo del vocabulario, los niños también fueron divididos en distintos niveles de acuerdo con el tamaño del vocabulario receptivo y expresivo (ver apartado de codificación en método).

De acuerdo con la revisión anterior, realizamos las siguientes predicciones: (1) los niños con SD mostrarán un mayor retraso en el desarrollo de la AC (producirán conductas menos maduras por lo que alcanzarán menores puntuaciones en $\mathrm{AC}$ ) que los niños con DT y (2) como consecuencia, los niños con SD tendrán un menor vocabulario (menor número de palabras) tanto en comprensión como en producción.

\section{Método}

\section{Participantes}

En el estudio participaron 131 niños con SD y 131 niños con DT de 8 a 29 meses de EM emparejados en EM y sexo. Los niños fueron divididos en siete grupos de edad mental (ver tabla 1). Todos los niños con SD mostraban trisomía-21 comprobada mediante documentación citogenética (los niños con mosaicismo y translocaciones fueron excluidos). Se excluyeron todos los niños con SD y todos los niños con DT con déficits neurosensoriales notorios (auditivos, visuales, etc.) y con claros problemas psicopatológicos. Todos los niños y sus familias eran hablantes nativos de español.

El contacto con las familias de niños con SD se realizaba a través de los Centros de Atención Temprana y Asociaciones de padres de niños con síndrome de Down de diferentes provincias españolas (principalmente del Sur). Los niños con DT del grupo control procedían de diferentes guarderías públicas y privadas de la ciudad de Málaga y sus alrededores. Todas las familias fueron informadas de los objetivos de la investigación y firmaron su consentimiento informado. La investigación se ajustó a las 
normas éticas de la Sociedad Española de Psicología.

Tabla 1. Media y desviación típica (entre paréntesis) de edad cronológica (EC) y edad mental (EM), ambas expresadas en meses, de los niños con SD y los niños con DT en cada nivel de edad mental.

\begin{tabular}{lllllll}
\hline $\begin{array}{l}\text { Nivel } \\
\text { EM }\end{array}$ & Grupo & Niñas & Niños & Total & $\begin{array}{l}\text { EM } \\
\text { Media (desviación típica) }\end{array}$ & \begin{tabular}{l} 
Media (desviación típica) \\
\hline $8-10$
\end{tabular} \\
& DS & 11 & 10 & 21 & $9,18(0,94)$ & $15,66(3,05)$ \\
& TD & 11 & 10 & 21 & $9,28(1,04)$ & $8,95(1,98)$ \\
$11-13$ & DS & 8 & 9 & 17 & $12,32(0,79)$ & $21,45(4,15)$ \\
& TD & 8 & 9 & 17 & $12,41(0,85)$ & $12,43(1,27)$ \\
$14-16$ & DS & 9 & 8 & 17 & $15,10(0,94)$ & $25,43(6,42)$ \\
& TD & 9 & 8 & 17 & $15,11(1,05)$ & $14,77(1,42)$ \\
$17-19$ & DS & 9 & 12 & 21 & $18,11(0,81)$ & $28,73(4,52)$ \\
& TD & 9 & 12 & 21 & $18,08(0,83)$ & $17,23(1,87)$ \\
$20-22$ & DS & 14 & 7 & 21 & $20,84(0,78)$ & $38,04(10,76)$ \\
& TD & 14 & 7 & 21 & $20,80(0,85)$ & $21,19(2,03)$ \\
$23-25$ & DS & 6 & 11 & 17 & $24,17(0,89)$ & $42,05(7,98)$ \\
& TD & 6 & 11 & 17 & $24,22(0,98)$ & $24,28(2,84)$ \\
$26-29$ & DS & 7 & 10 & 17 & $27,47(0,95)$ & $56,56(8,18)$ \\
& TD & 7 & 10 & 17 & $27,46(0,96)$ & $26,88(2,91)$ \\
TOTAL & DS & 64 & 67 & 131 & $17,98(6,05)$ & $32,09(14,45)$ \\
& TD & 64 & 67 & 131 & $18,00(6,03)$ & $17,76(6,33)$ \\
\hline
\end{tabular}

\section{Materiales}

Para la recogida de datos se empleó: (1) la adaptación del Inventario de Desarrollo Comunicativo MacArthur-Bates al perfil evolutivo de los niños con SD (CDI-Down) y (2) la adaptación realizada por Hernández (1995) del cuestionario sobre "evaluación de la comunicación" (ECO). Por lo que respecta al CDI-Down, se tuvo en cuenta los ítems del apartado de gestos y acciones relativos a la AC. En cuanto al ECO, sólo se consideró algunos ítems que complementaban la información obtenida a través del inventario. Los ítems concretos empleados son expuestos en el siguiente apartado.

La EM fue evaluada a través de la Escala de Desarrollo Psicomotor de la Primera Infancia Brunet-Lézine-Revisada (Josse, 1997).

Codificación de las respuestas 
Los ítems del Inventario y del ECO considerados fueron clasificados en diferentes categorías de AC. Más concretamente, las categorías e ítems empleados fueron los siguientes (I: inventario, E: ECO):

\section{Categoría 1: Conductas protoimperativas}

I-1. Extiende su mano, a veces acompañado de un gemido u otro sonido, para pedir algo I-2. A veces, también pide algo extendiendo la mano y abriéndola y cerrándola (es como si dijera 'dame')

I-3. Trata de que usted haga algo con algún objeto o juguete dándoselo o señalándoselo (por ejemplo, le señala la televisión para que la encienda, o le da el cochecito para que lo haga funcionar)

\section{Categoría 2: Conductas protodeclarativas}

I-4. Extiende su brazo para mostrarle / darle algo que tiene en la mano

I-5. Trata de alcanzar algún juguete u objeto para dárselo o enseñárselo

I-6. Señala (con el dedo índice extendido) algún objeto o situación interesantes para llamar su atención sobre ellos

\section{Categoría 3: Mirada al socio comunicativo en conductas protodeclarativas}

I-7. En los casos anteriores, también le mira a usted a la cara o a los ojos (ej.: abre y cierra la mano y le mira a la cara)

\section{Categoría 4: Uso del lenguaje en la interacción}

E- petición de objeto: Si su hijo/a ve algo que quiere coger y que no puede alcanzar, ¿Cómo te lo hace saber? (únicamente la conducta: 'Señala y dice algo así como dame'). E- denominación: Si hijo/a ve algo interesante ¿Cómo se refiere a ello? (únicamente la conducta: 'Dice algo así como mira').

E- Inicia la interacción: ¿Qué hace hijo/a para iniciar la interacción? (únicamente la conducta: 'Llama por el nombre').

Para estimar que un niño concreto dominaba una determinada categoría, los padres tenían que haber respondido 'muchas veces' o 'ya no lo hace' (en este último caso, el nivel del niño sería más maduro) en al menos dos de las tres conductas de las categorías 1 y 2 . En la categoría 3 debían darse esas mismas respuestas para la conducta 
examinada (en todas estas categorías, no se contabilizó la respuesta 'a veces' por considerar que en este caso la conducta no estaría claramente adquirida). En la categoría 4 debía responderse positivamente a las 3 conductas para asegurar que el niño tenía ya una cierta competencia lingüística.

Se concedía un punto a cada niño por cada categoría de AC manifestada, desde la menos (protoimperativas) a la más compleja (uso del lenguaje en la interacción). Estas puntuaciones iban sumándose según se iban acumulando categorías. Por ejemplo, si un niño realizaba conductas protoimperativas, se le concedía un punto. Si realizaba conductas protoimperativas + protodeclarativas, se le concedía dos puntos, etc. Si no realizaba aún conductas de $\mathrm{AC}$ de las definidas anteriormente, se le calificaba con un cero. De este modo, la muestra quedó dividida en 5 niveles de AC: (1) todavía no muestra conductas de AC, (2) conductas protoimperativas, (3) conductas protodeclarativas, (4) protodeclarativas con mirada al socio comunicativo y (5) uso del lenguaje en la interacción. Hay que hacer notar que la categoría "no realización de conductas de AC" se refiere a las conductas examinadas en este trabajo. De este modo, los niños pueden realizar otras conductas relacionadas con la $\mathrm{AC}$ que no han sido evaluadas, tales como seguir los gestos de otra persona, responder a sus peticiones de $\mathrm{AC}$, etc.

Para analizar la relación entre nivel de AC y tamaño del vocabulario, se dividió el vocabulario receptivo y el expresivo en diferentes niveles en función del número de palabras comprendidas o producidas. Más concretamente, los niveles fueron los siguientes para la producción léxica: nivel $1=<10$ palabras, nivel $2=11-50$, nivel $3=$ $51-100$, nivel $4=101-250$, nivel $5=251-400$ y nivel $6=>400$. Los primeros niveles se escogieron porque los puntos de corte de 10 y 50 palabras suelen ser considerados hitos en el desarrollo del lenguaje. El resto de niveles se seleccionaron de modo que reflejaran la evolución del vocabulario productivo. Por lo que respecta a la comprensión, los niveles escogidos fueron los siguientes: nivel 1=1-100 palabras, nivel $2=101-200$, nivel $3=201-300$, nivel $4=301-400$, nivel $5=401-500$ y nivel $6=>501$. Estos niveles se eligieron de modo que reflejaran la evolución del vocabulario receptivo (dado que la comprensión es superior a la producción, el rango de puntuaciones es mayor en este caso). 


\section{Procedimiento}

Se realizaron dos entrevistas. Estas entrevistas eran individuales o en pequeños grupos (no más de 5 familias). En la primera entrevista se explicaba los objetivos de la investigación, así como las características del inventario y el contenido de las diferentes secciones. Cuando los padres entregaban el inventario, los examinadores lo revisaban cuidadosamente en otra entrevista individual a fin de comprobar que se había rellenado correctamente. La tarea de los padres consistía en marcar aquellos gestos o conductas que sus hijos realizaban.

\section{Resultados}

En la tabla 2, puede observarse el número de niños con SD y niños con DT en cada una de las categorías de AC. Para comprobar el grado de relación entre ambas variables, se realizó un análisis de Chi-cuadrado para cada nivel de edad mental y para el total. Ninguno de ellos resultó significativo, aunque estos resultados hay que considerarlos con precaución dado que un número elevado de casillas (a excepción del análisis global) mostraba valores esperados inferiores a 5 . Si se tienen en cuenta los residuos corregidos (puntuaciones superiores o inferiores a $\pm 1,96$ son significativas al nivel de 0,05 ), como puede apreciarse en la tabla 2, apenas si existen diferencias significativas. Estos resultados muestran que no existen diferencias entre los niños con SD y los niños con DT por lo que respecta a las distintas conductas de AC examinadas, tanto si se consideran las distintas edades individualmente, como si se hace globalmente.

Por lo que respecta al vocabulario, en la tabla 3 puede observarse las medias de palabras obtenidas por los niños con SD y con DT en cada uno de los niveles de AC en comprensión y producción oral. Con el fin de comprobar las posibles diferencias entre las distintas variables y niveles dentro de cada una, se realizó un ANOVA mixto de medidas repetidas y factores entre-sujetos. Los factores entre-sujetos y los niveles dentro de cada uno de ellos fueron: factor Condición (SD y DT) y factor Nivel de AC (no conductas de AC, protoimperativos, protodeclarativos, protodeclarativos con mirada al socio comunicativo y uso del lenguaje en la interacción). El factor de medidas repetidas fue el Tipo de vocabulario, con dos niveles: comprensión y producción oral. Como nivel alfa se considero el valor de 0,05 . 
Tabla 2. Número de niños con SD y niños con DT en cada uno de los niveles de AC para cada nivel de EM (en meses) y globalmente (entre paréntesis se expresan los residuos corregidos

\begin{tabular}{|c|c|c|c|c|c|c|c|}
\hline \multirow[b]{2}{*}{$\begin{array}{l}\text { Nivel } \\
\text { de EM }\end{array}$} & \multirow[b]{2}{*}{ Grupo } & \multicolumn{6}{|c|}{ Nivel de $\mathrm{AC}$} \\
\hline & & $\begin{array}{l}\text { Todavía } \\
\text { no }\end{array}$ & Protoimperativos & Protodeclarativos & $\begin{array}{l}\text { Protodeclarativos } \\
\text { con mirada }\end{array}$ & $\begin{array}{l}\text { Uso de } \\
\text { lenguaje }\end{array}$ & Total \\
\hline \multirow{2}{*}{$8-10$} & SD & $18(1,4)$ & $0(-2,1) *$ & $2(1,4)$ & $1(-1,1)$ & 0 & 21 \\
\hline & DT & $14(-1,4)$ & $4(2,1) *$ & $0(-1,4)$ & $3(1,1)$ & 0 & 21 \\
\hline \multirow{2}{*}{$11-13$} & SD & $7(1,5)$ & $1(0,0)$ & $4(0,0)$ & $5(-1,4)$ & 0 & 17 \\
\hline & DT & $3(-1,5)$ & $1(0,0)$ & $4(0,0)$ & $9(1,4)$ & 0 & 17 \\
\hline \multirow{2}{*}{$14-16$} & $\mathrm{SD}$ & $5(0,0)$ & $0(-1,8)$ & $1(0,0)$ & $10(0,7)$ & $1(1,0)$ & 17 \\
\hline & DT & $5(0,0)$ & $3(1,8)$ & $1(0,0)$ & $8(-0,7)$ & $0(-1,0)$ & 17 \\
\hline \multirow{2}{*}{$17-19$} & SD & $3(-0,4)$ & $3(0,0)$ & $3(0,5)$ & $10(-0,6)$ & $2(1,4)$ & 21 \\
\hline & DT & $4(0,4)$ & $3(0,0)$ & $2(-0,5)$ & $12(0,6)$ & $0(-1,4)$ & 21 \\
\hline \multirow{2}{*}{$20-22$} & $\mathrm{SD}$ & $0(-1,8)$ & $2(1,4)$ & $2(-0,5)$ & $15(1,0)$ & $2(-0,5)$ & 21 \\
\hline & DT & $3(1,8)$ & $0(-1,4)$ & $3(0,5)$ & $12(-1,0)$ & $3(0,5)$ & 21 \\
\hline \multirow{2}{*}{$23-25$} & $\mathrm{SD}$ & $0(-1,0)$ & $3(1,8)$ & $3(0,5)$ & $6(-0,7)$ & $5(-0,4)$ & 17 \\
\hline & DT & $1(1,0)$ & $0(-1,8)$ & $2(-0,5)$ & $8(0,7)$ & $6(0,4)$ & 17 \\
\hline \multirow{2}{*}{$26-29$} & SD & $0(-1,5)$ & $0(-1,5)$ & $5(2,4) *$ & $6(0,0)$ & $6(-0,4)$ & 17 \\
\hline & $\mathrm{DT}$ & $2(1,5)$ & $2(1,5)$ & $0(-2,4) *$ & $6(0,0)$ & $7(0,4)$ & 17 \\
\hline \multirow{2}{*}{ Total } & SD & $33(0,1)$ & $9(-0,9)$ & $20(1,5)$ & $53(-0,6)$ & $16(0,0)$ & 131 \\
\hline & DT & $32(-0,1)$ & $13(0,9)$ & $12(-1,5)$ & $58(0,6)$ & $16(0,0)$ & 131 \\
\hline
\end{tabular}

Tabla 3. Medias obtenidas en cada uno de los niveles de AC en comprensión y producción por los niños con SD y con DT (desviación típica y número de niños entre paréntesis).

\begin{tabular}{|c|c|c|c|c|c|c|c|}
\hline \multirow[b]{2}{*}{$\begin{array}{l}\text { Tipo de } \\
\text { vocabulario }\end{array}$} & \multirow[b]{2}{*}{ Condición } & \multicolumn{6}{|c|}{ Nivel de AC } \\
\hline & & $\begin{array}{l}\text { Todavía } \\
\text { no }\end{array}$ & Protoimperativos & Protodeclarativos & $\begin{array}{l}\text { Protodeclarativos } \\
\text { con mirada }\end{array}$ & $\begin{array}{l}\text { Uso de } \\
\text { lenguaje }\end{array}$ & Total \\
\hline \multirow{3}{*}{ Comprensión } & SD & $\begin{array}{l}80,03 \\
(87,96)\end{array}$ & $\begin{array}{l}264,55 \\
(109,21)\end{array}$ & $\begin{array}{l}320,85 \\
(215,95)\end{array}$ & $\begin{array}{l}296,28 \\
(153,23)\end{array}$ & $\begin{array}{l}440,62 \\
(145,00)\end{array}$ & $\begin{array}{l}261,08 \\
(186,05)\end{array}$ \\
\hline & DT & $\begin{array}{l}90,78 \\
(121,85)\end{array}$ & $\begin{array}{l}186,85 \\
(192,66)\end{array}$ & $\begin{array}{l}198,67 \\
(159,17)\end{array}$ & $\begin{array}{l}261,29 \\
(159,59)\end{array}$ & $\begin{array}{l}439,69 \\
(162,43)\end{array}$ & $\begin{array}{l}228,30 \\
(184,97)\end{array}$ \\
\hline & Total & $\begin{array}{l}85,32 \\
(105,30)\end{array}$ & $\begin{array}{l}218,64 \\
(165,18)\end{array}$ & $\begin{array}{l}275,03 \\
(202,94)\end{array}$ & $\begin{array}{l}278,00 \\
(156,86)\end{array}$ & $\begin{array}{l}440,16 \\
(151,46)\end{array}$ & $\begin{array}{l}244,66 \\
(185,88)\end{array}$ \\
\hline \multirow{3}{*}{ Producción } & SD & $\begin{array}{l}4,51 \\
(7,13)\end{array}$ & $\begin{array}{l}80,00 \\
(80,87)\end{array}$ & $\begin{array}{l}140,95 \\
(183,93)\end{array}$ & $\begin{array}{l}92,39 \\
(148,48)\end{array}$ & $\begin{array}{l}293,62 \\
(206,99)\end{array}$ & $\begin{array}{l}101,39 \\
(162,35)\end{array}$ \\
\hline & DT & $\begin{array}{l}21,34 \\
(49,71)\end{array}$ & $\begin{array}{l}99,69 \\
(186,05)\end{array}$ & $\begin{array}{l}60,08 \\
(86,06)\end{array}$ & $\begin{array}{l}98,62 \\
(151,15)\end{array}$ & $\begin{array}{l}302,19 \\
(161,15)\end{array}$ & $\begin{array}{l}101,18 \\
(155,32)\end{array}$ \\
\hline & Total & $\begin{array}{l}12,8 \\
(35,98)\end{array}$ & $\begin{array}{l}91,64 \\
(149,56)\end{array}$ & $\begin{array}{l}110,62 \\
(157,94)\end{array}$ & $\begin{array}{l}95,65 \\
(149,23)\end{array}$ & $\begin{array}{l}297,91 \\
(182,53)\end{array}$ & $\begin{array}{l}101,29 \\
(158,57)\end{array}$ \\
\hline
\end{tabular}


En el ANOVA resultaron significativos los factores vocabulario y nivel de AC, así como las interacciones Vocabulario x Nivel de AC y Condición x Vocabulario. En el caso del factor Vocabulario: $F(1,252)=363,14, p<0,000$, eta cuadrado parcial $=0,59$. Como puede comprobarse en la tabla 3, la media total para comprensión es superior a la de producción oral. En cuanto al factor Nivel de AC: $F(4,252)=30,47, p<0,000$, eta cuadrado parcial $=0,326$. Comparados dos a dos los distintos niveles de este factor mediante Scheffé, se encontró que las medias obtenidas en 'todavía no' eran inferiores al resto, no existiendo diferencias entre las medias de 'protoimperativos', 'protodeclarativos' y 'protodeclarativos con mirada'. Todas los niveles anteriores eran inferiores al 'nivel uso del lenguaje'. De este modo, la secuencia encontrada es la siguiente: todavía no $<$ protoimperativos $=$ protodeclarativos $=$ protodeclarativos con mirada $<$ uso del lenguaje.

Los resultados anteriores quedan matizados si tenemos en cuenta las interacciones significativas encontradas. Por lo que respecta a la interacción Vocabulario x Nivel de AC: $F(4,252)=13,16, p<0,000$, eta cuadrado parcial = 0,173. Analizados los efectos simples de esta interacción (ajuste de la probabilidad mediante Bonferroni), se encontró que en todos los niveles de AC, la comprensión fue superior a la producción oral (ver en la tabla 2 el total para producción y comprensión en cada nivel de AC). La interacción Condición x Vocabulario también resultó significativa: $F(1,252)=7,22, p<0,008$, eta cuadrado parcial $=0,028$. Analizados los efectos simples de esta interacción (ajuste de la probabilidad mediante Bonferroni), se encontró que los niños con SD mostraban una puntuación superior en comprensión en relación con los niños con DT, mientras que en producción oral no existían diferencias (ver total para producción y comprensión en niños con SD y niños con DT en la tabla 2). No obstante, puesto que la eta cuadrado parcial no es muy elevada para esta interacción, este resultado hay que tomarlo con cautela.

El factor condición, la interacción Condición x Nivel de AC y la triple interacción Condición x Vocabulario x Nivel de AC no resultaron significativas. De este modo, no existían diferencias entre los niños con SD y DT tomadas todas las puntuaciones en conjunto. De igual modo, los niños con SD y con DT se comportaban de forma parecida en cada nivel de AC. Este resultado nos muestra que no existen diferencias entre ambos grupos por lo que respecta a los niveles de AC y su relación con el vocabulario.

Un aspecto a resaltar es la alta variabilidad individual que se observa tanto en los 
resultados obtenidos por los niños con SD como por los niños con DT. En consecuencia, es posible que la puntuación alcanzada por un niño particular en una categoría de $\mathrm{AC}$ menos madura podría ser similar al obtenido por otro en otra categoría más madura.

Aunque el análisis anterior es importante, nos deja sin responder cómo va evolucionando el nivel de AC en función del tamaño del vocabulario. Para intentar dar una respuesta a esta cuestión, se dividió el vocabulario receptivo y el expresivo en diferentes niveles en función del número de palabras comprendidas o producidas. Como se indicó en el apartado de codificación (ver método), los niveles para producción fueron los siguientes: nivel $1=<10$ palabras, nivel $2=11-50$, nivel $3=51-100$, nivel 4 $=101-250$, nivel $5=251-400$ y nivel $6=>400$. Por lo que respecta a la comprensión, los niveles escogidos fueron: nivel $1=1-100$ palabras, nivel $2=101-200$, nivel $3=201$ 300 , nivel $4=301-400$, nivel $5=401-500$ y nivel $6=>501$.

En la tabla 4 puede observarse los resultados obtenidos para cada nivel de producción y comprensión en función del nivel de AC de los niños con SD y con DT. Como puede comprobarse en dicha tabla, la semejanza entre los niños con SD y los niños con DT es muy elevada tanto para comprensión como para producción. Esto se hace más patente si observamos las columnas y filas del total de niños. Si tenemos en cuenta el cruce entre las distintas variables (casillas), también se observa una gran similitud. Para determinar el grado de relación entre el nivel de AC y el nivel de vocabulario se realizaron cuatro análisis de Chi-cuadrado, dos para comprensión y dos para producción (en cada caso, uno para el grupo de niños con SD y otro para el grupo de niños con DT). Todos resultaron significativos, aunque hay que considerarlos con precaución dado que un elevado número de casillas mostraban valores esperados inferiores a 5. Por lo que respecta a la producción, los resultados fueron los siguientes: Chi-cuadrado $=83,82$, g.1. 20, $\mathrm{p}<0,000$ en el caso del grupo con SD, y Chi-cuadrado= 72,68 , g.1. $20, \mathrm{p}<0,000$ en el caso del grupo con DT. Por lo que respecta a la comprensión: Chi-cuadrado=83,61, g.1. 20, p $<0,000$ en el caso del grupo con SD y Chi-cuadrado $=68,51$, g.l. 20, $\mathrm{p}<0,000$ en el caso del grupo con DT. Ello significa que ambas variables no son independientes sino que, por el contrario, guardan una relación entre ellas. 
Tabla 4. Número de niños con SD y con DT teniendo en cuenta los niveles de atención conjunta y de vocabulario receptivo y expresivo (entre paréntesis se expresan los residuos corregidos)

\begin{tabular}{|c|c|c|c|c|c|c|c|}
\hline \multirow[b]{2}{*}{$\begin{array}{l}\text { Nivel } \\
\text { vocabulario }\end{array}$} & \multirow[b]{2}{*}{ Condición } & \multicolumn{6}{|c|}{ Nivel de AC } \\
\hline & & Todavía no & Protoimperativos & Protodeclarativos & $\begin{array}{l}\text { Protodeclarativos } \\
\text { con mirada }\end{array}$ & $\begin{array}{ll}\text { Uso } & \mathrm{de} \\
\text { lenguaje } & \end{array}$ & Total \\
\hline & & \multicolumn{6}{|c|}{ Comprensión } \\
\hline \multirow{2}{*}{$1-100$} & SD & $25(7,2)^{*}$ & $1(-1,1)$ & $4(-0,8)$ & $6(-3,4)^{*}$ & $0(-2,6)^{*}$ & 36 \\
\hline & DT & $23(5,7)^{*}$ & $6(1,2)$ & $3(-0,5)$ & $9(-3,5)^{*}$ & $0(-2,9)^{*}$ & 41 \\
\hline \multirow{2}{*}{$101-200$} & SD & $5(-0,2)$ & $1(-0,4)$ & $4(0,5)$ & $10(0,7)$ & $1(-1,1)$ & 21 \\
\hline & DT & $4(-1,2)$ & $3(0,3)$ & $4(1,2)$ & $13(0,7)$ & $2(-0,8)$ & 26 \\
\hline \multirow{2}{*}{$201-300$} & SD & $1(-2,5)^{*}$ & $4(2,2)^{*}$ & $3(0,3)$ & $12(1,3)$ & $3(0,1)$ & 23 \\
\hline & DT & $2(-2,2)^{*}$ & $2(-0,4)$ & $2(-03)$ & $19(3,3)^{*}$ & $1(-1,5)$ & 26 \\
\hline \multirow{2}{*}{$301-400$} & SD & $1(-2,3)^{*}$ & $1(-04)$ & $2(-0,7)$ & $14(2,9)^{*}$ & $2(-0,3)$ & 20 \\
\hline & DT & $2(-0,5)$ & $0(-1,2)$ & $2(1,1)$ & $5(0,1)$ & $2(0,6)$ & 11 \\
\hline \multirow{2}{*}{$401-500$} & SD & $1(-1,2)$ & $2(1,7)$ & $0(-1,4)$ & $4(0,0)$ & $3(1,8)$ & 10 \\
\hline & DT & $0(-1,9)$ & $0(-1,1)$ & $0(-1,0)$ & $5(0,4)$ & $5(3,8)^{*}$ & 10 \\
\hline \multirow{2}{*}{$>501$} & SD & $0(-2,9)^{*}$ & $0(-1,4)$ & $7(2,5)^{*}$ & $7(-07)$ & $7(3,2)^{*}$ & 21 \\
\hline & DT & $1(-1,9)$ & $2(0,3)$ & $1(-0,5)$ & $7(-0,3)$ & $6(3,1)^{*}$ & 17 \\
\hline \multirow{2}{*}{ Total } & SD & 33 & 9 & 20 & 53 & 16 & 131 \\
\hline & DT & 32 & 13 & 12 & 58 & 16 & 131 \\
\hline & & \multicolumn{6}{|c|}{ Producción } \\
\hline \multirow{2}{*}{$<10$} & SD & $31(7,0)^{*}$ & $2(-1,2)$ & $7(-0,7)$ & $13(-3,0)^{*}$ & $1(-3,1)^{*}$ & 55 \\
\hline & DT & $24(5,1)^{*}$ & $7(1,3)$ & $5(0,3)$ & $9,9(-3,2)^{*}$ & $0(-3,3)^{*}$ & 49 \\
\hline \multirow{2}{*}{$11-50$} & SD & $2(-2,7)^{*}$ & $3(0,8)$ & $3(0,9)$ & $20(3,3)^{*}$ & $2(-1,1)$ & 30 \\
\hline & DT & $3(-2,3)^{*}$ & $2(0,8)$ & $4(0,8)$ & $22(3,2)^{*}$ & $1(-1,8)$ & 32 \\
\hline \multirow{2}{*}{$51-100$} & SD & $0(-2,2)^{*}$ & $1(0,1)$ & $3(0,8)$ & $7(1,0)$ & $2(0,4)$ & 13 \\
\hline & DT & $4(0,4)$ & $1(-04)$ & $0(-1,3)$ & $8(1,0)$ & $1(-0,6)$ & 14 \\
\hline \multirow{2}{*}{$101-250$} & SD & $0(-2,0)^{*}$ & $3(2,8)^{*}$ & $1(06)$ & $5(0,4)$ & $2(0,6)$ & 11 \\
\hline & DT & $1(-1,7)$ & $1(-0,4)$ & $3(1,5)$ & $6(-0,4)$ & $4(1,8)$ & 15 \\
\hline \multirow{2}{*}{$251-400$} & SD & $0(-1,9)$ & $0(-0,9)$ & $4(2,3)^{*}$ & $3(-0,7)$ & $3(1,8)$ & 10 \\
\hline & DT & $0(-1,8)$ & $0(-1,0)$ & $0(-1,0)$ & $4(0,0)$ & $5(4,1)^{*}$ & 9 \\
\hline \multirow{2}{*}{$>400$} & SD & $0(-2,1)^{*}$ & $0(-1,0)$ & $2(0,1)$ & $4(-0,5)$ & $6(4,2)^{*}$ & 12 \\
\hline & DT & $0(-2,1)^{*}$ & $2(0,8)$ & $0(-1,2)$ & $5(-0,2)$ & $5(3,3)^{*}$ & 12 \\
\hline \multirow{2}{*}{ Total } & SD & 33 & 9 & 20 & 53 & 16 & 131 \\
\hline & DT & 32 & 13 & 12 & 58 & 16 & 131 \\
\hline
\end{tabular}

Significativo al 0.05

Con el fin de comprobar el significado de esa relación, se hallaron los residuos corregidos en cada una de las casillas (las puntuaciones superiores o inferiores a $\pm 1,96$ son significativas al nivel de 0,05$)$. Como puede observarse en la tabla 4 , los resultados significativos fueron escasos, pero muestran un patrón interesante (hay que tener en 
cuenta que puntuaciones positivas indican que se han hallado más casos de los esperados y puntuaciones negativas lo contrario). Dicho patrón, de nuevo, es bastante similar por lo que respecta a los niños con SD y con DT y tanto para comprensión como para producción. De este modo, se aprecia que en los primeros niveles de vocabulario predomina la categoría todavía no, la cual va disminuyendo según aumenta el número de palabras producidas / comprendidas por los niños. El patrón contrario se encuentra por lo que respecta a la categoría uso del lenguaje. Exceptuando estos patrones, lo único que habría que destacar es la preponderancia de las categorías de protodeclarativos y protodeclarativos con mirada, si bien no siempre resultaron significativas.

\section{Discusión}

Los resultados obtenidos no confirman la primera de nuestras predicciones al no encontrar diferencias entre los niños con SD y los niños con DT por lo que respecta a las distintas categorías de AC examinadas. Este resultado es bastante potente en la medida en que aparecía no solo cuando se consideraban todos los datos globalmente, sino también para cada nivel de EM. Tan solo habría que destacar que los niños con SD producían un número algo mayor de conductas protodeclarativas y algo menos protoimperativas, aunque las diferencias no llegaban a ser significativas tomando todos los datos en conjunto. Esto apoyaría los resultados de Mundy et al. (1988), quienes encontraron que las conductas imperativas les suponen un mayor esfuerzo que las declarativas a estos niños.

Algo similar ocurre por lo que respecta al vocabulario. De este modo, a diferencia de lo que habíamos predicho, los niños con SD no producen ni comprenden menos palabras que los niños con DT. Incluso en el caso de la comprensión, su vocabulario es superior. El mismo resultado fue obtenido por Galeote, Sebastián, Checa, Rey y Soto (2011) y es consistente con el perfil evolutivo de los niños con SD quienes presentan una mejor actuación en comprensión que en producción en general. De hecho, la comprensión suele ser considerada un punto fuerte en esta población (CardosoMartins, Mervis y Mervis, 1985; Caselli, Vicari, Longobardi, Lami, Pizzoli y Stella, 1998; Chapman, Schwartz y Kay-Raining Bird, 1991; Galeote et al., 2011). Una posible explicación es su mayor experiencia de vida a causa de su mayor edad cronológica (Chapman, 1995, 2006).

Por otro lado, teniendo en cuenta las distintas categorías de AC en función de los diferentes niveles léxico, el parecido entre los niños con SD y los niños con DT es 
notable, apareciendo el mismo perfil. Por esta razón, los comentarios que siguen se refieren a ambos grupos de niños. La categoría todavía no, de cierta frecuencia en el primer nivel de vocabulario, iba disminuyendo según aumentaba el número de palabras producidas o comprendidas por los niños. La categoría de uso del lenguaje, mostraba el patrón contrario.

Los resultados en el resto de categorías muestran también que existen pocas diferencias. Teniendo en cuenta los distintos niveles de vocabulario, aparecía una preponderancia (aunque no siempre significativa) de las categorías protodeclarativas y protodeclarativas con mirada. Ello revelaría, por un lado, que aunque en el primer nivel de vocabulario (tanto receptivo como expresivo) los niños muestran variadas conductas de AC, aun cuando predomine la categoría todavía no, en el siguiente nivel y posteriores los niños comienzan a emplear todas las categorías de AC, a excepción de uso del lenguaje que, como hemos visto, es más tardía (no hay que olvidar que, al ser acumulativas las puntuaciones, obtener una puntuación en protodeclarativas y protodeclarativas con mirada implica que también se realizan conductas protoimperativas). Por otro lado, este resultado apoyaría que en torno a los 12 meses los niños comienzan a emplear prácticamente todas las categorías de $\mathrm{AC}$, si bien hay que esperar algún tiempo para que empleen el lenguaje en las interacciones (Carpenter et al., 1998).

El resultado anterior también podría explicar el patrón encontrado en el ANOVA sobre comprensión y producción: todavía no $<$ protoimperativos $=$ protodeclarativos $=$ protodeclarativos con mirada $<$ uso del lenguaje. Como podemos comprobar, hay dos saltos importantes. El primero se da cuando los niños comienzan a producir conductas de AC. Esto apoyaría la propuesta de Bates et al. (1989) de que las habilidades de interacción social y el lenguaje expresivo comparten una varianza común en el área de la motivación social y la iniciación de conductas sociales. Quizás pueda proponerse algo similar para el lenguaje receptivo: para interaccionar con los otros también hay que aprender palabras. Sin embargo, esto no sería suficiente para explicar el segundo salto, cuando comienzan a usar de forma predominante el lenguaje en sus interacciones. Este resultado, no obstante, encontraría su justificación en la medida en que en el desarrollo del lenguaje expresivo se hallan implicados otros factores que van más allá de lo meramente interactivo, tales como la maduración de los órganos de articulación y el desarrollo fonológico (Leonard, Schwartz, Morris y Chapman, 1981) o la habilidad para recuperar palabras de la memoria léxica (ver Gershkoff-Stowe, Thal, Smith y Namy, 
1997, para una discusión sobre el tema). De este modo, la producción de conductas interactivas, incluidas las implicadas en contextos de AC, serviría como plataforma para que los niños se sientan motivados a aprender el lenguaje de los que les rodean, pero no es suficiente en sí misma.

Una cuestión importante tiene que ver con el supuesto estilo directivo de interacción de las madres de niños con SD. Como se vio en la introducción, diversos trabajos han encontrado que las madres de niños con SD son más directivas y controladoras (Cardoso-Martins y Mervis, 1985; Mahoney et al., 1990; Tannock, 1988), cambian de tópico más frecuentemente y lo controlan más (Mahoney et al., 1990; Tannock, 1988), independientemente de que los niños estén o no implicados con los objetos (Mahoney et al., 1990; Maurer y Sherrod, 1987; Tannock, 1988), etc. Pese a ello, algunos autores proponen que estas conductas serían una consecuencia de su adaptación al nivel de competencia de sus hijos (ver Galeote, Checa, Serrano y Rey, 2004, para una revisión).

Los resultados anteriores son relevantes en la medida en que se basan en una amplia muestra de niños. Pese a ello, existen algunas limitaciones relacionadas con el empleo de informes paternos. En estos informes, sólo se tiene acceso a la información relativa a la presencia / ausencia de una determinada conducta, pero desconocemos aspectos cruciales tales como: frecuencia de los episodios de AC, su duración, el tipo de habla que emplean los cuidadores en dichos episodios, etc. (existen, no obstante, algunos datos que apuntan a que no existen diferencias en estos factores -Galeote, Checa, Sánchez, Gamarro y Fernández, 2012). Además, dadas las características del CDI-Down, no se han tenido en cuenta todas las conductas implicadas en la AC. Nuestro análisis se ha basado exclusivamente en conductas de AC iniciadas por el niño. Algunos autores defienden que la habilidad de los niños para responder a conductas de AC o seguir la mirada de los otros puede reflejar el desarrollo de importantes habilidades sociales, cognitivas y auto-regulatorias relacionadas con el desarrollo del lenguaje (Baldwin y Baird, 1999; Moore y Corkum, 1994; Tomasello, 1995). De hecho, como se indicó en la introducción, varios estudios han señalado que las diferencias individuales en la habilidad de los niños de 6 a 18 meses para responder a la AC son predictivas de las habilidades lingüísticas entre los 24 y 36 meses (Markus, Mundy, Morales, Delgado y Yale, 2000; Morales, Mundy y Rojas, 1998; Mundy y Gomes, 1998; Mundy et al., 1995).

Partiendo de los resultados obtenidos sería interesante comprobar en futuras 
investigaciones la relación entre los estilos comunicativos de los adultos, las funciones comunicativas empleadas por los niños y los procesos de $\mathrm{AC}$, así como su incidencia en el desarrollo lingüístico y comunicativo posterior.

\section{Bibliografía}

Akhtar, N.; Dunham, F. \&Dunham, P.J. (1991). Directive interactions and early vocabulary development: the role of joint attentional focus. Journal of Child Language, 18, 41-49.

Bakeman, R. \& Adamson, L. (1986). Infant's conventionalized acts: gestures and words with mothers and peers. Infant Behavior and Development, 9, 215-230.

Baldwin, D. \& Baird, J. (1999). Action analysis: A gateway to intentional inference. En P. Rochat (Ed.), Early social cognition: Understanding others in the first months of life (pp. 215-240). Mahwah, NJ: LEA.

Bates, E., Benigni, L., Bretherton, I., Camaioni, L. \& Volterra, V. (1979). The emergency of symbols: Cognition and communication in infancy. New York: Academic Press.

Bates, E., Thal, D., Fenson, L., Whitesell, K. \& Oakes, L. (1989). Integrating language and gesture in infancy. Developmental Psychology, 25, 1004-1019.

Beeghly, M., Perry. B.W. \& Cicchetti, D. (1989). Structural and affective dimensions of play development in young children with Down syndrome. International Journal of Behavioral Development, 12, 257-277.

Cardoso-Martins, C. \& Mervis C.B. (1985). Maternal speech to prelinguistic children with Down syndrome. American Journal on Mental Deficiency, 89, 451-458.

Cardoso-Martins, C., Mervis C.B. \& Mervis, C.A. (1985). Early vocabulary acquisition by children with Down syndrome. American Journal on Mental Deficiency, 90, 177-184.

Carpenter, M., Nagel, K. \& Tomasello, M. (1998). Social cognition, joint attention and communicative competence from 9 to 15 months of age. Monographs of the Society for Research in Child Development, 63 (4), serial n 255.

Caselli, M.C., Vicari, S., Longobardi, E., Lami, L., Pizzoli, C. \& Stella, G. (1998). Gestures and words in early development of children with Down syndrome. Journal of Speech, Language and Hearing Research, 41, 1125-1135.

Chapman, R. S. (1995). Language development in children and adolescents with Down Syndrome. En P. Fletcher, \& B. MacWhinney (Eds.), The handbook of child 
language (pp. 641-663). Oxford, UK: Blackwell.

Chapman, R. S. (2006). Language learning in Down syndrome: The speech and language profile compared to adolescents with cognitive impairment of unknown origin. Down Syndrome Research \& Practice, 10, 61-66.

Chapman, R., Schwartz, S.E. \& Kay-Raining Bird, E. (1991). Language skills of children and adolescents with Down Syndrome: I. Comprehension. Journal of Speech and Hearing Research, 34, 1106-1120.

Cielinski, K.L., Vaughn, B.E., Seifer, R. \& Contreras, J. (1995). Relations among sustained engagement during play, quality of play, and mother-child interaction in samples of children with Down syndrome and normally developing toddlers. Infant Behavior and Development, 18, 163-176.

Davis, H., Stroud, A. \& Green, L. (1988). Maternal language environment of children with mental retardation. American journal on mental retardation, 93, 144-153.

Fischer, M.A. (1987). Mother-child interaction in preverbal children with Down Syndrome. Journal of Speech and Hearing Disorders, 52, 179-190.

Franco, F. \& Wishart, J.G. (1995). The use of pointing and other gestures by young children with Down syndrome. American Journal on Mental Retardation, 100, 160-182.

Galeote, M., Checa, E., Sánchez, C., Gamarro, A. y Fernández, E. (2012). Atención conjunta y desarrollo del vocabulario en niños con síndrome de Down. Comunicación presentada en el XVIII Congreso Internacional de la Asociación Española de Logopedia, Foniatría y Audiología, julio, Madrid.

Galeote, M., Checa, E., Serrano, A. y Rey, R. (2004). De la evaluación a la intervención: atención conjunta, directividad y desarrollo del lenguaje en niños con síndrome de Down. Revista Síndrome de Down, 21, 114-121.

Galeote, M., Sebastián, E., Checa, E., Rey, R. \& Soto, P. (2011). The development of vocabulary in Spanish children with Down syndrome: Comprehension, production and gestures. Journal of Intellectual \& Developmental Disability, 36, 184-96.

Galeote, M., Soto, P., Lamela, E., Checa, E., Pulido, L. y Rey, R. (2006). Adaptación de los Inventarios de Desarrollo Comunicativo MacArthur-Bates para el estudio del desarrollo del lenguaje en niños con síndrome de Down. Revista de Atención Temprana, 9, 4-16.

Gershkoff-Stowe, L., Thal, D.J., Smith, L.B. y Namy, L.L. (1997). Categorization and 
its developmental relation to early language. Child Development, 68, 843-859.

Harris, M., Jones, D., Brookes, S. \& Grant, J. (1986). Relations between the non-verbal context of maternal speech and rate of language development. British Journal of Developmental Psychology, 4, 261-268.

Hernández, J.M. (1995). Propuesta curricular en el área de lenguaje: Educación Infantil y alumnos con trastornos de la comunicación. Madrid: CEPE.

Josse, D. (1997). Escala de desarrollo psicomotor de la primera infancia Brunet -Lézine-Revisado (edición española). Madrid: Psymtéc.

Kasari, C., Freeman, S., Mundy, P. \& Sigman, M.D. (1995). Attention regulation in children with Down syndrome: coordinated joint attention and social referencing. American Journal on Mental Deficiency, 100, 128-136.

Krakow, J.B. \& Koop, C.B. (1983). The effects of developmental delay on sustained attention in young children. Child Development, 54, 1143-1155.

Laakso, M., Poikkeus, A.M., Katajamäki, J. \& Lyytinen, P. (1999). Early intentional communication as a predictor of language development in young toddlers. First Language, 19, 207-231.

Landry, S. \& Chapieski, M.L. (1989). Joint attention and infant toy exploration: effects of Down syndrome and prematurity. Child Development, 60, 103-118.

Legerstee, M. \& Bowman, T.G. (1989). The development of responses to people and a toy in infants with Down syndrome. Infant Behavior and Development, 12, 465477.

Legerstee, M. \& Weintraub, J. (1997). The integration of person and object attention in infants with and without Down syndrome. Infant behavior and development, 20, 71-82.

Leonard, L., Schwartz, R., Morris, B. y Chapman, K. (1981). Factors influencing early lexical acquisition: lexical orientation and phonological composition. Child Development, 52, 882-887.

MacTurk, R.H., Vietze, P.M., McCarthy, M.E., McQuiston, S. \& Yarrow, L.J. (1985). The organization of exploratory behavior in Down syndrome and nondelayed children. Child Development, 56, 573-581.

Mahoney, G., Fors, S. \& Wood, S. (1990). Maternal directive behavior revisited. American Journal on Mental Deficiency, 94, 398-406.

Markus, J., Mundy, P., Morales, M., Delgado, C.E. \& Yale, M. (2000). Individual differences in infant skill as predictors of child-caregiver joint attention and 
language. Social Development, 9, 302-315.

Maurer, H. \& Sherrod, K. (1987). Context of directives given to young children with Down syndrome and non retarded children: development over two years. American Journal on Mental Deficiency, 91, 579-590.

Moore, C. \& Corkum, V. (1994). Social understanding at the end of the first year of life. Developmental Review, 14, 349-372.

Morales, M., Mundy, P. \& Rojas, J. (1998). Gaze following and language development in six-month-olds. Infant Behavior and Development, 21, 373-377.

Mundy, P. \& Gomes, A. (1998). Individual differences in joint attention skill development in second year. Infant Behavior and Development, 21, 469-482.

Mundy, P., Kasari, C., Sigman, M. \& Ruskin, E. (1995). Nonverbal communication and early language acquisition in children with Down syndrome and in normally developing children. Journal of Speech and Hearing Research, 38, 157-167.

Mundy, P., Sigman, M., Kasari, C. \& Yirmiya, N. (1988). Nonverbal communication skills in Down syndrome children. Child Development, 59, 235-249.

Seibert, J., Hogan, A. \& Mundy, P. (1982). Assessing interactional competencies: The early social-communication scales. Infant Mental Health Journal, 3, 244-258.

Sigman, M. \& Ruskin, E. (1999). Continuity and change un the social competence of children with autismo, Down syndrome, and developmental delays. Monographs of the Society for Research in Child Development, $\mathrm{n}^{\mathrm{o}} 64$ (1, serial number 256).

Tannock, R. (1988). Mother's directiveness in their interactions with their children with and without Down syndrome. American Journal on Mental Deficiency, 93, 154165.

Tomasello, M. (1995). Join attention as social cognition. En C. Moore y P. Dunham (Eds.), Joint attention: Its origin and role in development (pp. 103-130). Hillsdale, NJ: LEA.

Tomasello, M. \& Farrar, M. (1986). Joint attention and early language. Child Development, 57, 454-463.

Tomasello, M. \& Todd, J. (1983). Joint attention and lexical acquisition style. First Language, 4, 197-212.

Trevarthen, C. (1998) The concept and foundations of infant intersubjetivity. En S. Braten (Ed.), Intersubjetive communication and emotion in early ontogeny. Cambridge: Cambridge University Press. 